\title{
EMPREGO DE BIOMARCADORES METABÓLICOS SALIVARES PARA O RASTREAMENTO DO CÂNCER BUCAL: REVISÃO DE LITERATURA
}

\author{
Anildo Alves de Brito Júnior ${ }^{1}$ \\ Jeisielle Alves da Anunciação Barreto ${ }^{2}$ \\ Marcelo Victor Coelho Marques ${ }^{1}$ \\ Myllena Miranda Silva ${ }^{1}$ \\ Márcia Otto Barrientos ${ }^{1}$ \\ Ivair Tavares Júnior ${ }^{1}$
}

${ }^{1}$ Faculdade Adventista da Bahia - FADBA. Cachoeira, BA, Brasil. Rodovia BR 101, km 197, Capoeiruçu, Cachoeira BA, Brasil.

2Programa Multiprofissional em Saúde da Família e Comunidade, Fundação Oswaldo Cruz (Fiocruz - Bahia). Instituto Gonçalo Moniz. Av. Waldemar Falcão, 121, Candeal - Salvador - BA, Brasil.

\section{RESUMO}

Introdução: O câncer bucal é o sexto tipo de câncer mais frequente, sendo sua alta taxa de mortalidade intrinsecamente relacionada ao diagnóstico tardio. A saliva como um fluido oral multiconstituinte, compreende secreções amplamente fornecidas pelo sangue, com potencial para detecção de biomarcadores específicos relacionados ao câncer bucal. Logo, o presente trabalho busca discutir o emprego de biomarcadores metabólicos salivares para o rastreamento e diagnóstico precoce do câncer bucal. Métodos: Trata-se de uma revisão integrativa da literatura, desenvolvida por meio da análise retrospectiva de estudos primários que avaliaram o papel de biomarcadores salivares para o rastreamento do câncer bucal. A base de dados PubMed foi consultada, utilizando os descritores: "biomarkers" AND "saliva" AND "oral cancer". Foram selecionados 17 estudos publicados nos últimos 5 anos, sem restrições de idioma. Resultados: O contato direto da saliva com lesões de câncer oral a torna uma ferramenta de rastreamento mais específica e potencialmente sensível, sendo registrada na literatura uma gama de biomarcadores salivares (DNA, RNA, mRNA, marcadores de proteína), incluindo citocinas (IL-8, IL-1b, TNF - $\alpha$ ), defensina-1, P53, Cyfra 21-1, antígeno específico de polipeptídeo de tecido, fosfatase de especificidade dupla, espermidina / espermina N1 acetil-transferase, profilina-1, cofilina-1 e transferrina. Esses metabólitos salivares combinados são considerados assinaturas moleculares e potenciais indicadores do processo patológico, podendo ser a base de um método clinicamente viável de rastreamento não invasivo do câncer oral. Conclusões: A abordagem combinada de biomarcadores salivares pode ser usada como ferramenta de triagem para melhorar a deteç̧ão precoce e a precisão do diagnóstico de câncer bucal.

Palavras-chave: Câncer bucal. Marcadores biológicos. Saliva.

\footnotetext{
*Autor correspondente: Anildo Alves de Brito Júnior. Faculdade Adventista da Bahia - FADBA, Cachoeira, BA, Brasil. Rodovia BR 101, km 197, Capoeiruçu, Cachoeira - BA, 44300-000. Tel: +55 (27) 98167-6098. E-mail: junioranildo02@gmail.com.
} 


\section{INTRODUÇÃO}

O câncer oral é considerado um tumor maligno da cavidade oral, sendo o sexto tipo de câncer mais comum em todo o mundo, com um incidente anual de 550.000 novos casos ${ }^{1}$. É responsável por $4 \%$ dos cânceres em homens e $2 \%$ dos cânceres em mulheres, levando ao óbito, anualmente, cerca de 380.000 indivíduos ${ }^{1,2}$. O carcinoma de células escamosas oral (CEC), também chamado de carcinoma epidermóide, representa $90 \%$ de todos os cânceres que acometem a região da cabeça e pescoço. Esse tumor tem uma alta taxa de recorrência e frequentemente sofre metástases em linfonodos ${ }^{3}$.

Apesar do progresso com estratégias preventivas e terapêuticas, 0 atraso no diagnóstico do CEC continua sendo uma das principais causas da alta morbimortalidade ${ }^{2}$. Cristaldi et al $(2019)^{2}$ relataram que a maior taxa de CEC é identificada em estágio clínico avançado (estágio III ou IV) e após o tratamento primário, recorrências e/ou metástases são encontradas em $80 \%$ dos casos nos primeiros 2 anos, com taxa de sobrevida em 5 anos inferior a $50 \%$. Atualmente, a investigação histológica através de biópsia representa o "padrão ouro" do diagnóstico de CEC, no entanto, essas análises podem não refletir a heterogeneidade tumoral. Estudos recentes avaliaram o uso potencial de métodos não invasivos, como biópsia líquida ${ }^{4}$.

Os tumores liberam várias biomoléculas nos fluidos corporais que podem ser utilizadas como biomarcadores para diagnóstico, prognóstico e seleção da terapia. As biópsias líquidas fornecem informações sobre as características moleculares durante a evolução do tumor por meio de uma abordagem não invasiva ${ }^{4}$. Nesse sentido, o desenvolvimento de modelos de agrupamento integrativo combinando dados moleculares de DNA, RNA como microRNAs (miRNAs/miRs) e caracterização de proteínas poderia fornecer maior precisão na compreensão da heterogeneidade do tumor, auxiliando no estabelecimento de estratégias terapêuticas específicas ${ }^{3-5}$.
A saliva como um fluido oral

multiconstituinte, compreende secreções amplamente fornecidas pelo sangue, com potencial para detecção de biomarcadores específicos relacionados ao câncer bucal. Javaid e colaboradores $(2016)^{6}$ em seu estudo, relataram que os níveis salivares de proteínas específicas apresentavam-se elevados em pacientes com CEC. Os níveis de CD44 (glicoproteína de superfície celular envolvida na interação intercelular), Cyfra 21-1 (fragmento de citoqueratina 19), antígeno de polipeptídeo de tecido (TPS) e antígeno de câncer 125 (CA125), por exemplo, foram sugeridos como biomarcadores de câncer oral detectados no fluido salivar ${ }^{6}$.

O uso de RNAs identificados na saliva (transcriptomas) associados ao CEC mostrou uma taxa de precisão de $81 \%$, demonstrando seu potencial como biomarcadores para a detecção do câncer oral, bem como para isolar indivíduos que podem desenvolver a doença ${ }^{5-6}$.

Dessa forma, o objetivo do presente trabalho é discutir, através de uma revisão integrativa da literatura, o emprego de biomarcadores metabólicos salivares para o rastreamento e diagnóstico precoce do câncer bucal.

\section{MÉTODOS}

Trata-se de um estudo descritivo, de caráter exploratório, desenvolvido através de uma revisão crítica e integrativa da literatura. Inicialmente, foi realizada uma análise retrospectiva de estudos primários que avaliaram o papel de biomarcadores salivares para o rastreamento do câncer bucal, através de consultas na base de dados da PubMedl MEDLINE. Os descritores DeCS/MeSH empregados durante a busca literária foram: "biomarkers", "saliva", "oral cancer".

O processo de seleção dos artigos e busca eletrônica aconteceu dentro de um período pré-determinado, entre outubro de 2020 a fevereiro de 2021. Após a seleção das palavras-chave, realizou-se o cruzamento na BVS (Biblioteca Virtual em Saúde) com o operador booleano AND: "biomarkers" AND "saliva" AND "oral cancer".

Para o refinamento da busca, os critérios de inclusão foram: artigos com textos 
completos que abordassem a temática proposta, publicações realizadas nos últimos 5 anos e sem restrições de idioma, sendo incluídas revisões narrativas, integrativas e sistemáticas da literatura, meta-análises, estudos experimentais em animais e ensaios clínicos. Já os critérios de exclusão foram: artigos em que o texto completo não estava disponível, estudos in vitro, monografias, dissertações e teses, além de trabalhos que não estavam relacionados ao tema proposto ou que estivessem fora do período de publicação pré-estabelecido. Foram encontrados 60 estudos iniciais, sendo 17 utilizados nesta revisão.

Após seleção dos estudos, realizou-se a leitura dos títulos, resumos e textos na íntegra. A triagem dos artigos foi desenvolvida por uma dupla de revisores, previamente calibrados, de forma independente e a inclusão final de todos os estudos foi desenvolvida após concordância. Os dados obtidos foram agrupados utilizando as ferramentas do Excel 2016 (Microsoft, Redmond WA).

\section{RESULTADOS}

Para esta revisão de literatura foram selecionados e analisados 17 trabalhos indexados na base de dados PubMed, publicados nos últimos 5 anos, que correspondiam aos objetivos da pesquisa e estavam de acordo com os critérios de inclusão e exclusão previamente determinados. No Quadro 1 foram caracterizados os estudos selecionados:

Quadro 1: Caracterização da produção científica analisada, de acordo com primeiro autor (ano), objetivo (s), métodos e resultados. Cachoeira- BA, Brasil, 2021.

\begin{tabular}{|c|c|c|c|}
\hline $\begin{array}{l}\text { AUTOR } \\
\text { (ANO) }\end{array}$ & OBJETIVO(S) & MÉTODOS & RESULTADOS \\
\hline 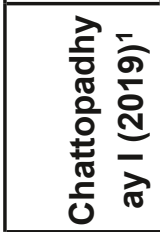 & $\begin{array}{l}\text { Descrever o papel do microbioma na } \\
\text { progressão do câncer bucal e seu } \\
\text { papel como biomarcador diagnóstico } \\
\text { e prognóstico precoce. }\end{array}$ & $\begin{array}{l}\text { Uma revisão narrativa da } \\
\text { literatura foi desenvolvida } \\
\text { com } 115 \text { artigos. }\end{array}$ & $\begin{array}{l}\text { Vários nichos de câncer oral foram } \\
\text { ocupados predominantemente por } \\
\text { bactérias, produzindo biomarcadores } \\
\text { identificados na saliva. }\end{array}$ \\
\hline 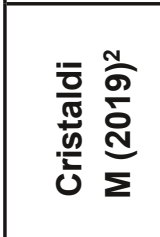 & $\begin{array}{l}\text { Relatar os dados mais recentes sobre a } \\
\text { detecção de biomarcadores de saliva } \\
\text { em biópsia de saliva líquida de } \\
\text { pacientes com câncer oral. }\end{array}$ & $\begin{array}{l}\text { Uma revisão narrativa da } \\
\text { literatura foi desenvolvida } \\
\text { com } 124 \text { artigos. }\end{array}$ & $\begin{array}{l}\text { A biópsia líquida de saliva tem vários } \\
\text { usos clínicos promissores no } \\
\text { tratamento do câncer bucal. É uma } \\
\text { alternativa indolor, acessível e de } \\
\text { baixo custo. }\end{array}$ \\
\hline 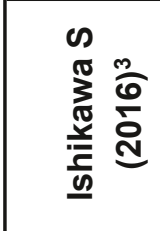 & $\begin{array}{l}\text { Explorar biomarcadores de } \\
\text { metabólitos salivares, traçando o } \\
\text { perfil de amostras da saliva e do tecido } \\
\text { tumoral. }\end{array}$ & $\begin{array}{l}\text { Amostras salivares de } 68 \\
\text { indivíduos divididos em } \\
\text { grupo controle e câncer oral } \\
\text { foram submetidas a análise } \\
\text { metabolômica. }\end{array}$ & $\begin{array}{l}85 \text { metabólitos mostraram diferenças } \\
\text { significativas entre o tumor e as } \\
\text { amostras de controle pareadas, e } \\
\text { entre as amostras salivares de câncer } \\
\text { oral e controles. }(p<0,05)\end{array}$ \\
\hline 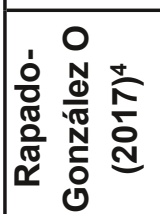 & $\begin{array}{l}\text { Fornecer aspectos da aplicação } \\
\text { clínica potencial de miRNAs livres de } \\
\text { células como biomarcadores do CEC. }\end{array}$ & $\begin{array}{lrr}\text { Revisão da } & \text { literatura } \\
\text { desenvolvida } & \text { com } 107 \\
\text { artigos. } & & \end{array}$ & $\begin{array}{l}\text { Os perfis de miRNA livres de células } \\
\text { representam uma abordagem não } \\
\text { invasiva promissora para o } \\
\text { diagnóstico de câncer oral. }\end{array}$ \\
\hline 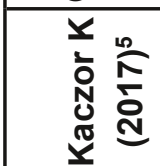 & $\begin{array}{l}\text { Fornecer uma atualização sobre as } \\
\text { aplicações atuais e futuras da saliva } \\
\text { para fins de diagnóstico. }\end{array}$ & $\begin{array}{l}\text { Revisão da } \quad \text { literatura } \\
\text { desenvolvida } \\
\text { com } 197 \\
\text { artigos. }\end{array}$ & $\begin{array}{l}\text { Avanços recentes ampliaram a } \\
\text { abordagem do diagnóstico salivar. }\end{array}$ \\
\hline 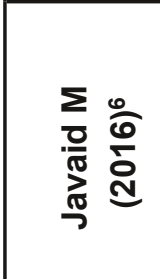 & $\begin{array}{l}\text { Descrever os avanços em } \\
\text { biomarcadores salivares para } \\
\text { diagnosticar doenças autoimunes, } \\
\text { doenças cardiovasculares, diabetes, } \\
\text { HIV, câncer oral, cárie e doenças } \\
\text { periodontais. }\end{array}$ & $\begin{array}{l}\text { Revisão da literatura } \\
\text { desenvolvida com } 71 \\
\text { artigos. }\end{array}$ & $\begin{array}{l}\text { Devido à sua facilidade e } \\
\text { acessibilidade não invasiva, } \\
\text { juntamente com sua abundância de } \\
\text { biomarcadores, como material } \\
\text { genético e proteínas, a saliva é uma } \\
\text { ferramenta de diagnóstico potencial. }\end{array}$ \\
\hline
\end{tabular}




\begin{tabular}{|c|c|c|c|}
\hline $\begin{array}{c}\text { AUTOR } \\
\text { (ANO) }\end{array}$ & OBJETIVO(S) & MÉTODOS & RESULTADOS \\
\hline $\begin{array}{l}\text { I } \\
\text { ลิ } \\
\text { ป }\end{array}$ & $\begin{array}{l}\text { Identificar um painel de } \\
\text { marcadores que mostra alta } \\
\text { sensibilidade e especificidade } \\
\text { para o diagnóstico de CEC. }\end{array}$ & 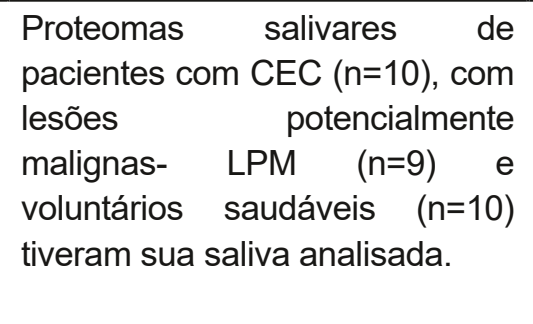 & $\begin{array}{l}\text { Os níveis salivares de } 67 \text { e } 18 \\
\text { proteínas no grupo CEC estão mais } \\
\text { elevados. O nível salivar mais alto do } \\
\text { fator do complemento } \mathrm{H} \text {, cadeia alfa do } \\
\text { fibrinogênio e alfa-1-antitripsina foi } \\
\text { relacionado com estágios avançados } \\
\text { do CEC. }\end{array}$ \\
\hline ఖ & $\begin{array}{l}\text { Investigar potenciais biomarcadores } \\
\text { na saliva e no plasma humanos } \\
\text { para auxiliar no diagnóstico precoce } \\
\text { do CEC. }\end{array}$ & $\begin{array}{l}\text { Amostras de saliva e plasma } \\
\text { obtidas de pacientes com CEC } \\
(n=41) \text { e pacientes com câncer } \\
\text { não oral } \quad(n=24) \text { foram } \\
\text { analisadas. }\end{array}$ & $\begin{array}{l}\text { IL-1 } 1 \beta \text {, IL-6, IL-8, MIP- } 1 \beta \text {, eotaxina e } \\
\text { IFN- } Y \text { e TNF- } \alpha \text { apresentaram } \\
\text { diferenças entre pacientes com CEC } \\
\text { e controles. }\end{array}$ \\
\hline 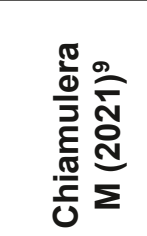 & $\begin{array}{l}\text { Descrever citocinas salivares com } \\
\text { potenciais } \quad \text { biomarcadores } \\
\text { diagnósticos para CEC. }\end{array}$ & $\begin{array}{l}\text { Revisão sistemática e meta- } \\
\text { análise de } 28 \text { artigos (de } 2004 \text { a } \\
\text { 2018) disponíveis na PubMed e } \\
\text { Cochrane Library. }\end{array}$ & $\begin{array}{l}\text { Os níveis salivares de algumas citocinas } \\
\text { são consistentemente diferentes entre } \\
\text { indivíduos com CEC. }\end{array}$ \\
\hline 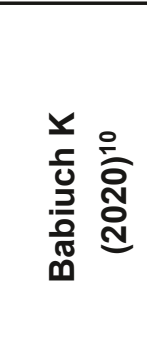 & $\begin{array}{l}\text { Avaliar a IL-1alfa, IL-6, IL-8 e TNF- } \\
\text { a em amostras de tecido e saliva } \\
\text { de pacientes com CEC e lesões } \\
\text { potencialmente malignas (LPM). }\end{array}$ & $\begin{array}{l}\text { As citocinas foram avaliadas em } \\
60 \text { amostras de tecido de lesões } \\
\text { patológicas (CEC ou LPM) e em } 7 \\
\text { controles (mucosa oral normal) } \\
\text { por imunohistoquímica e na saliva } \\
\text { de } 45 \text { pacientes com CEC ou } \\
\text { LPM e } 9 \text { controles. }\end{array}$ & $\begin{array}{l}\text { A análise imunohistoquímica } \\
\text { revelou expressão significativamente } \\
\text { maior de IL-8 e TNF- } \alpha \text { em } \\
\text { espécimes e amostras salivares de } \\
\text { CEC e LPM, com displasia em } \\
\text { comparação com mucosa normal. }\end{array}$ \\
\hline 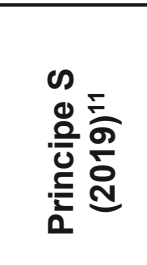 & $\begin{array}{l}\text { Analisar o papel dos elementos } \\
\text { da resposta inflamatória salivar } \\
\text { em pacientes com CEC tratados } \\
\text { com radioterapia. }\end{array}$ & $\begin{array}{l}15 \text { artigos publicados nos } \\
\text { últimos } 10 \text { anos, na PubMed, } \\
\text { Cochrane e Embase, foram } \\
\text { analisados. }\end{array}$ & $\begin{array}{l}\text { O tratamento radioterápico gerou } \\
\text { uma tendência de crescimento dos } \\
\text { níveis de IL-1 } 1 \beta, \text { IL-6, IL-8, MCP-1 e } \\
\text { TNF- } \alpha \text {, que se correlacionou com a } \\
\text { dose de irradiação. }\end{array}$ \\
\hline 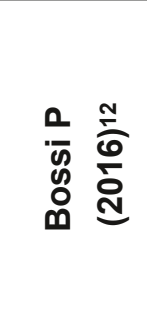 & $\begin{array}{l}\text { Avaliar a presença de citocinas } \\
\text { salivares e sua modulação } \\
\text { durante a terapia oncológica } \\
\text { associando com a gravidade da } \\
\text { mucosite oral. }\end{array}$ & $\begin{array}{ll}\text { Os níveis salivares de } & 13 \\
\text { citocinas foram analisados em } \\
55 \text { pacientes com CEC, } 10 \\
\text { voluntários saudáveis e } 10 \\
\text { pacientes com outros tipos de } \\
\text { câncer. }\end{array}$ & $\begin{array}{l}\text { Os níveis de citocinas aumentaram } \\
\text { durante o tratamento, com aumento } \\
\text { de interleucina- } 1 \beta \quad(p=0,009) \text {, IL- } 6 \\
(p=0,005) \text { e fator de necrose } \\
\text { tumoral- } \alpha(p=0,005) \text {. }\end{array}$ \\
\hline 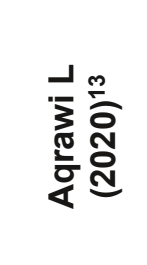 & $\begin{array}{l}\text { Investigar como os efeitos tardios } \\
\text { da radioterapia podem influenciar } \\
\text { os perfis de citocinas. }\end{array}$ & $\begin{array}{l}\text { Análise de perfis de citocinas } \\
\text { na saliva e fluido lacrimal de } 29 \\
\text { pacientes com câncer irradiado } \\
\text { de cabeça e pescoço e } 20 \\
\text { controles. }\end{array}$ & $\begin{array}{l}\text { As citocinas elevadas identificadas } \\
\text { na saliva dos pacientes com câncer } \\
\text { foram: CCL21, IL-4, CX3CL1, } \\
\text { CCL2, CXCL1 e CCL15. }(p<0,01)\end{array}$ \\
\hline 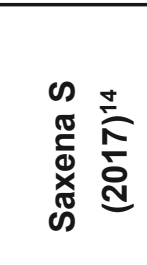 & $\begin{array}{l}\text { Identificar os biomarcadores } \\
\text { potenciais para a triagem e a } \\
\text { análise de patologia molecular em } \\
\text { pacientes de alto risco com CEC. }\end{array}$ & $\begin{array}{l}\text { Revisão sistemática de } 50 \\
\text { trabalhos indexados nas bases } \\
\text { de dados PubMed, EBSCO, } \\
\text { Cochrane, Science Direct, ISI } \\
\text { web Science e SciELO. }\end{array}$ & $\begin{array}{l}\text { A proteína de ligação Mac- } 2 \text {, } \\
\text { proteína } 14 \text { relacionada ao mieloide, } \\
\text { CD59, profilina } 1 \text { e catalase estavam } \\
\text { em níveis elevados em pacientes } \\
\text { com câncer oral. }\end{array}$ \\
\hline
\end{tabular}




\begin{tabular}{|c|c|c|c|}
\hline $\begin{array}{l}\text { AUTOR } \\
\text { (ANO) }\end{array}$ & OBJETIVO(S) & MÉTODOS & RESULTADOS \\
\hline 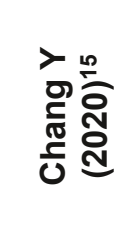 & $\begin{array}{l}\text { Analisar os níveis de MMP-1 na } \\
\text { saliva de indivíduos saudáveis e } \\
\text { pacientes com lesões } \\
\text { potencialmente malignas (LPM) ou } \\
\text { CEC. }\end{array}$ & $\begin{array}{l}\text { Mediu-se o conteúdo de MMP-1 } \\
\text { em amostras de saliva de } 1160 \\
\text { indivíduos ( } 313 \text { controles } \\
\text { saudáveis, } 578 \text { com LPM e } 269 \\
\text { pacientes com CEC). }\end{array}$ & $\begin{array}{l}\text { Os níveis salivares de MMP-1 } \\
\text { discriminaram significativamente os } \\
\text { pacientes com CEC de grupos não } \\
\text { cancerosos. }\end{array}$ \\
\hline ح & $\begin{array}{l}\text { Avaliar a atividade da IL-6 em } \\
\text { pacientes com CEC. }\end{array}$ & $\begin{array}{l}\text { Uma revisão sistemática foi } \\
\text { desenvolvida analisando } 127 \\
\text { artigos indexados na PubMed } \\
\text { entre } 1995 \text { e } 2019 \text {. }\end{array}$ & $\begin{array}{l}\text { As concentrações séricas de IL-6 e } \\
\text { a expressão de IL-6 são candidatos } \\
\text { a biomarcadores de prognóstico. }\end{array}$ \\
\hline m & $\begin{array}{l}\text { Validar o potencial diagnóstico de } \\
\text { microRNAs (miRNAs) por meio da } \\
\text { análise de amostras de biópsia } \\
\text { líquida obtidas de } 10 \text { pacientes com } \\
\text { CEC e } 10 \text { controles saudáveis. }\end{array}$ & $\begin{array}{l}\text { A expressão de miRNAs } \\
\text { selecionados foi avaliada em } \\
\text { uma coorte piloto de dez } \\
\text { pacientes com câncer oral e dez } \\
\text { doadores saudáveis. }\end{array}$ & $\begin{array}{l}\text { Os níveis de expressão dos preditos hsa- } \\
\text { miR-133a-3p e hsa-miR-375-3p } \\
\text { regulados negativamente foram } \\
\text { significativamente reduzidos em } \\
\text { pacientes com câncer oral. }(p<0,001)\end{array}$ \\
\hline
\end{tabular}

\section{DISCUSSÃO}

A saliva é um fluido corporal complexo que contém diversos tipos de biomoléculas, incluindo uma ampla variedade de enzimas, hormônios, anticorpos, constituintes antimicrobianos e fatores de crescimento ${ }^{6}$. Sua constituição é descrita como um ultrafiltrado do sangue, já que, a maioria das moléculas identificas na corrente sanguínea são também detectadas na saliva. Através de vias transcelulares (transporte passivo ou ativo), vias paracelulares (ultrafiltração extracelular) ou do sulco gengival, as mais diversas biomoléculas do organismo passam a constituir o fluido salivar ${ }^{3-7}$.

Em pacientes com CEC as células cancerosas e outros constituintes do microambiente tumoral são circundadas pelo meio salivar, possibilitando a detecção de marcadores salivares para a triagem. A literatura tem relatado mais de 100 moléculas salivares como potenciais biomarcadores do CEC, incluindo proteínas, nucleotídeos (DNA, mRNA e microRNA) e metabólitos ${ }^{7}$. Esses marcadores podem ser detectados antes mesmo que O CEC seja identificado clinicamente ou ainda nos casos de pacientes que apresentam lesões potencialmente malignas, permitindo que o diagnóstico e triagem de risco dos pacientes seja obtido de forma precoce ${ }^{8}$.

Algumas vantagens que tornam a saliva uma amostra emergente para o diagnóstico de doenças são: pode ser colhida facilmente e de forma econômica e não invasiva, podendo ser empregada para pesquisas epidemiológicas. Além disso, amostras salivares são fáceis de armazenar e transportar, não coagulam e podem refletir o estado fisiológico atual de um indivíduo ${ }^{5,13}$.

As células cancerosas liberadas da massa tumoral primária se disseminam por todos os fluidos corporais, podendo liberar ativamente fragmentos de ácidos nucleicos celulares (cfDNA), que, inclusive, quando internalizados por células não cancerosas, induzem a transformação neoplásica ${ }^{13-15}$. Em pacientes oncológicos, existe um prejuízo na remoção das moléculas derivadas de células, induzindo o acúmulo de cfDNA no microambiente do tecido e fluidos biológicos ${ }^{2}$. O ctDNA é liberado principalmente na corrente sanguínea, no entanto, também pode ser encontrado em outros fluidos corporais, especialmente na saliva. Através da ultrafiltração de glândula salivar, difusão passiva ou transporte ativo, o ctDNA pode facilmente atingir a saliva de um sítio local e corrente sanguínea, transportando informações sobre tumores primários e/ou metástases. Destaca-se que, em virtude da menor diluição e contaminação, a análise do ctDNA na saliva é muito mais sensível do que no sangue ${ }^{5}$.

O cfDNA liberado pelas células malignas 
pode ser distinguido do cfDNA fisiológico por meio de vários recursos que incluem a concentração de biofluido, mutações somáticas e tamanho geral. Dessa forma, uma análise do fluido salivar pode diferenciar os tipos de cfDNA, detectando a presença daqueles que estiverem alterados ${ }^{14}$.

Chang et al $(2020)^{15}$ identificaram que quando as principais alterações genéticas do CEC em ctDNA de saliva e amostras de tecido foram analisadas, uma correlação na perda de heterozigosidade e mutações de p53 foi encontrada, demonstrando que a saliva pode ser uma alternativa confiável e não invasiva à biópsia de tecido para detecção do câncer de cabeça e pescoço.

MicroRNAs são pequenas moléculas de RNA de fita simples (19-21 nucleotídeos), transcritas pelo RNA polimerase celular e submetidas a um corte sequencial no núcleo (miRNA primário) e a um corte no citoplasma (miRNA precursor). Essas moléculas, dependendo do nível de complementaridade com o alvo, têm o potencial de clivar um RNA mensageiro (mRNA) ou inibir sua tradução, o que as tornam reguladores de processos neoplásicos ${ }^{4}$.

Os MicroRNAs podem ser identificados na saliva como miRNAs livres de células, associados a proteínas de ligação ou seletivamente empacotados em vesículas. Rapado-González $(2017)^{4}$ cita em seu estudo 13 miRNAs significativamente desregulados em amostras de saliva de CEC oral em comparação com controles saudáveis, incluindo 11 miRNAs regulados para baixo (miR-136, miR-147, miR-1250, miR-148a, miR632, miR-646, miR-668, miR-877, miR-503, miR-220a e miR-323-5p) e dois miRNAs regulados positivamente (miR-24e miR-27b). $O$ envolvimento dessas moléculas em microambientes tumorais tem demonstrado que a expressão de miRNAs pode estar associada à desregulação de genes oncossupressores ou oncogenes, contribuindo para o desenvolvimento ou inibição do tumor.

Ao desenvolverem uma análise comparativa de biomediadores presentes na saliva de paciente com CEC e pacientes saudáveis, Aqrawi et al $(2020)^{13}$ evidenciaram níveis significativamente mais elevados de diversas citocinas (CCL21, CX3CL1, CXCL1, CCL2 e CCL15) no grupo de pacientes com CEC quando comparados a controles saudáveis, além de alterações nas vias de sinalização mediadas por interleucinas, sendo a IL-3 (60\%), IL-5 (60\%) e IL-23 (40\%) as mais alteradas, seguidas da IL-1 (20\%), IL-2 (20\%), , IL-4 $(20 \%)$ e IL-12 $(20 \%)^{13}$.

Outras interleucinas intrinsecamente relacionadas a processos oncológicos são a IL6 e IL-8. Tratam-se de citocinas próinflamatórias que desempenham um papel crucial em vários mecanismos de imunorregulação e tumorigênese, estando em concentrações mais elevadas na saliva de pacientes com CEC ${ }^{16}$.

O TNF- $\alpha$ tem sido relatado como potencial biomarcador de prognóstico para CEC que pode ser identificado através da análise do fluido salivar. O papel do TNF- $\alpha$ na regulação de processos tumorais é justificado pela sua capacidade de ativar as vias endógenas de ERK1/2 e NF-KB, induzindo a expressão de miR-450 ${ }^{a}$, consequentemente aumentando o grau de invasão das células malignas.

Chiamulera et al $(2021)^{9}$ descreve valores de TNF- $\alpha$ mais elevados em pacientes com CEC, sendo que, para os grupos controle foram identificados valores de 0,013 a $11.300,0$ $\mathrm{pg} / \mathrm{mL}$, enquanto que para pacientes com CEC, os valores de TNF- $\alpha$ variaram de 0,739 a $23.100,0 \mathrm{pg} / \mathrm{mL}$.

Outros biomarcadores alterados em cerca de $20 \%$ dos processos cancerosos e identificados no fluido salivar são TNF e TGF$\beta$. A via apoptótica do p53 (20\%) também foi influenciada por citocinas salivares reguladas positivamente 4 . A detecção de células tumorais circulantes (CTCs) liberadas da massa tumoral primária está correlacionada a metástases, recorrências e pior prognóstico da doença. As CTCs disseminam-se através dos vasos 
linfáticos e sanguíneos por todo o corpo, sendo que, estudos futuros sobre CTCs salivares de pacientes com CEC podem fornecer informações relevantes sobre o prognóstico do tumor e alterações genéticas potencialmente associadas à recorrência, contribuindo para o desenvolvimento de terapias direcionadas ${ }^{10-12}$.

\section{CONCLUSÃO}

A abordagem combinada de biomarcadores salivares pode ser usada como ferramenta de triagem para melhorar a detecção precoce e a precisão do diagnóstico de câncer bucal. $O$ desenvolvimento de mais estudos que envolvam a análise salivar auxiliarão para 0 estabelecimento de estratégias consistentes para o diagnóstico precoce de lesões cancerígenas, contribuindo para a prevenção avançada e apoiando o desenvolvimento de terapias direcionadas.

\section{AGRADECIMENTOS}

Aos colegas e professores do curso de Odontologia da Faculdade Adventista da Bahia (FADBA).

\section{CONFLITOS DE INTERESSES}

Os autores declaram que a pesquisa foi realizada na ausência de quaisquer conflitos de interesses.

\section{REFERÊNCIAS:}

1. Chattopadhyay I, Verma M, Panda M. Role of Oral Microbiome Signatures in Diagnosis and Prognosis of Oral Cancer. Technol Cancer Res Treat. 2019 Jan 1;18. doi: 10.1177/1533033819867354. PMID: 31370775; PMCID: PMC6676258.

2. Cristaldi M, Mauceri R, Di Fede O, Giuliana G, Campisi G, Panzarella V. Salivary Biomarkers for Oral Squamous Cell Carcinoma Diagnosis and Follow-Up: Current Status and Perspectives. Front Physiol. 2019 Dec; 10:1476. doi: 10.3389/fphys.2019.01476. PMID: 31920689; PMCID: PMC6914830.

3. Ishikawa $S$, Sugimoto $M$, Kitabatake $K$, Sugano A, Nakamura M, Kaneko $\mathrm{M}$ et al. Identification of salivary metabolomic biomarkers for oral cancer screening. Sci Rep. 2016 Aug 19; 6:315-20. doi: 10.1038/ srep31520. PMID: 27539254; PMCID: PMC4990923.

4. Rapado-González Ó, López-López R, López-Cedrún JL, Triana-Martínez G, MuineloRomay L, Suárez-Cunqueiro MM. Cell-Free microRNAs as Potential Oral Cancer Biomarkers: From Diagnosis to Therapy. Cells. 2019 Dec 17; 8(12):16-53. doi: 10.3390/ cells8121653. PMID: 31861130; PMCID: PMC6952938.

5. Kaczor-Urbanowicz KE, Martin CarrerasPresas C, Aro K, Tu M, Garcia-Godoy F, Wong DT. Saliva diagnostics - Current views and directions. Exp Biol Med (Maywood). 2017 Mar; 242(5):459-472. doi: 10.1177/1535370216681550. PMID: 27903834; PMCID: PMC5367650.

6. Javaid MA, Ahmed AS, Durand R, Tran SD. Saliva as a diagnostic tool for oral and systemic diseases. J Oral Biol Craniofac Res. 2016 JanApr; 6(1):66-75. doi: 10.1016/ j.jobcr.2015.08.006. PMID: 26937373; PMCID: PMC4756071.

7. Chu HW, Chang KP, Hsu CW, Chang IY, Liu HP, Chen YT et al. Identification of Salivary Biomarkers for Oral Cancer Detection with Untargeted and Targeted Quantitative Proteomics Approaches. Mol Cell Proteomics. 2019 Sep; 18(9):1796-1806. doi: 10.1074/ mcp.RA119.001530. PMID: 31253657; PMCID: PMC6731081.

8. Lee LT, Wong YK, Hsiao HY, Wang YW, Chan MY, Chang KW. Evaluation of saliva and plasma cytokine biomarkers in patients with oral squamous cell carcinoma. Int $\mathrm{J}$ Oral Maxillofac Surg. 2018 Jun; 47(6):699-707. doi: 10.1016/j.ijom.2017.09.016. PMID: 29174861.

9. Chiamulera MMA, Zancan CB, Remor AP, Cordeiro MF, Gleber-Netto FO, Baptistella AR. Salivary cytokines as biomarkers of oral cancer: a systematic review and meta-analysis. BMC Cancer. 2021 Feb 27; 21(1):205. doi: 10.1186/s12885-021-07932-3. 
33639868; PMCID: PMC7912500.

10. Babiuch $K$, Kuśnierz-Cabala $B$, Kęsek $B$, Okoń K, Darczuk D, Chomyszyn-Gajewska M. Evaluation of Proinflammatory, NF-kappaB Dependent Cytokines: IL-1 $\alpha$, IL-6, IL-8, and TNF- $\alpha$ in Tissue Specimens and Saliva of Patients with Oral Squamous Cell Carcinoma and Oral Potentially Malignant Disorders. J Clin Med. 2020 Mar 21; 9(3):867. doi: 10.3390/ jcm9030867. PMID: 32245251; PMCID: PMC7141524.

11. Principe S, Dikova V, Bagán J. Salivary Cytokines in patients with Head and Neck Cancer (HNC) treated with Radiotherapy. J Clin Exp Dent. 2019 Nov; 11(11):e1072-e1077. doi: 10.4317/jced.56318. PMID: 31700580; PMCID: PMC6825732.

12. Bossi P, Bergamini C, Miceli R, Cova A, Orlandi E, Resteghini $C$ et al. Salivary Cytokine Levels and Oral Mucositis in Head and Neck Cancer Patients Treated With Chemotherapy and Radiation Therapy. Int J Radiat Oncol Biol Phys. 2016 Dec 1; 96(5):959-966. doi: 10.1016/ j.jrobp.2016.08.047. PMID: 27745982.

13. Aqrawi LA, Chen X, Hynne H, Amdal C, Reppe S, Aass HCD et al. Cytokines Explored in Saliva and Tears from Radiated Cancer Patients Correlate with Clinical Manifestations, Influencing Important Immunoregulatory Cellular Pathways. Cells. 2020 Sep 8; 9(9):2050. doi: 10.3390/cells9092050. PMID: 32911805; PMCID: PMC7565699.

14. Saxena S, Sankhla B, Sundaragiri KS, Bhargava A. A Review of Salivary Biomarker: A Tool for Early Oral Cancer Diagnosis. Adv Biomed Res. 2017 Jul 28; 6:90. doi: 10.4103/2277-9175.211801. PMID: 28828341; PMCID: PMC5549541.

15. Chang YT, Chu LJ, Liu YC, Chen CJ, Wu $\mathrm{SF}$, Chen $\mathrm{CH}$ et al. Verification of Saliva Matrix Metalloproteinase-1 as a Strong Diagnostic Marker of Oral Cavity Cancer. Cancers (Basel). 2020 Aug 13; 12(8):2273. doi: 10.3390/ cancers12082273. PMID: 32823758; PMCID: PMC7463746.
16. Uz U, Eskiizmir G. Association Between Interleukin-6 and Head and Neck Squamous Cell Carcinoma: A Systematic Review. Clin Exp Otorhinolaryngol. $2021 \mathrm{Feb}$; 14(1):50-60. doi: 10.21053/ceo.2019.00906. PMID: 33587847; PMCID: PMC7904429.

17. Crimi S, Falzone L, Gattuso G, Grillo CM, Candido S, Bianchi A et al. Droplet Digital PCR Analysis of Liquid Biopsy Samples Unveils the Diagnostic Role of hsa-miR-133a-3p and hsamiR-375-3p in Oral Cancer. Biology (Basel). 2020 Nov 6; 9(11):379. doi: 10.3390/ biology9110379. PMID: 33172167; PMCID: PMC7694750. 